\title{
Christoph Moor
}

\section{»Ein so erklärtes Lieblingsstück der hiesigen Kunstfreunde«. \\ Die Rezeptionsgeschichte der Jupiter-Sinfonie in Beethovens Wien}

Als Ludwig van Beethoven im Jahr 1792 nach Wien kam, um bei Joseph Haydn zu studieren, lag die Entstehungszeit der Jupiter-Sinfonie gerade einmal vier Jahre zurück und Mozart war seit einem Jahr tot. Dass Beethoven die Werke Mozarts bereits zu diesem Zeitpunkt kannte und sogar eingehend studiert hatte, zeigt das Beispiel der Jupiter deutlich. Zwar ist nicht belegt, ob Beethoven eine Abschrift der Partitur oder das Stimmenmaterial der Sinfonie besaß, doch der Musikwissenschaftler Carl Schachter weist in seinem Artikel »Mozart's Last and Beethoven's First« auf die unübersehbaren Parallelen zwischen Mozarts Jupiter und dem ersten Satz von Beethovens Sinfonie Nr. I C-Dur op. 2I hin. ${ }^{\text {I }}$ Schachters Analyse des harmonischen Aufbaus der Durchführung und der markanten Modulationen lassen für ihn keinen Zweifel daran, dass Beethoven I799, zu Beginn der Arbeit an seiner Sinfonie, die Jupiter genau kannte. Dabei spielt es für ihn keine Rolle, ob Beethoven ein Notentext zur Verfügung stand; Schachter hält es für möglich, dass der Einfluss, den die Jupiter auf Beethoven ausübte, allein durch das Hören zustande kommen konnte: »Even if he had no access to a copy of the score, Beethoven would surely have been able to assimilate much of the music by ear; and he could have supplemented his listening by a perusal of the parts. $\varkappa^{2}$ Dabei stellt sich allerdings die Frage, ob Beethoven überhaupt die Möglichkeit hatte, die Jupiter mit Orchester zu hören - das Eingangszitat, das die Jupiter als »erklärtes Lieblingsstück« ausweist, bezieht sich, wie wir später sehen werden, auf Leipzig und nicht auf Wien.

Wir werden im Folgenden den gängigen Namen >Jupiter ‘ für diese Sinfonie verwenden, der erstmals I8I9 am Edinburgh-Festival in einer Kritik auftaucht. ${ }^{3}$ Vorher war das Werk, nicht zuletzt im deutschen Sprachraum, vor allem als >Sinfonie mit der Schlussfuge oder `Große Sinfonie in C mit der Fuge < bekannt. Erst der Konzertveranstalter Johann Peter Salomon in London gab der Sinfonie den Übernamen Jupiter, da er ihre Qualität und Überlegenheit manifestieren wollte. ${ }^{4}$ Zur Verbreitung dieses Titels trug

Vgl. Carl Schachter: Mozart's Last and Beethoven's First. Echoes of K. 55I in the First Movement of Opus 2I, in: Mozart Studies, hg. von Cliff Eisen, Bd. I, Oxford I99I, S. 227-25I.

2 Ebd., S.227.

3 Hyatt King wies den Namen Jupiter in einem Konzertprogramm des Edinburgh Music Festival vom 20. Oktober I819 nach; vgl. Hyatt King: Mozart in Retrospect. Studies in Criticism and Bibliographp, London I955, 2., rev. Ausg. 1956, S. 264.

4 H. C. Robbins Landon: Zum vorliegenden Band, in: Wolfgang Amadeus Mozart: Orchesterwerke. Sinfonien, Bd. 9, Wien 1957 (Neue Ausgabe sämtlicher Werke, Serie Iv, Werkgruppe II, Bd. 9), S. viI-XII, hier S. XII. 
später auch die in London angefertigte Bearbeitung der Jupiter für Klavier, Flöte, Geige und Cello von Muzio Clementi aus dem Jahr I823 bei; sie zeigt auf dem Titelblatt den Gott Jupiter in den Wolken thronend, Blitze in der rechten und ein Zepter in der linken Hand haltend. 5

Wien als Musikstadt In der Vorrede zu seinem Jahrbuch der Tonkunst von Wien und Prag von 1796 attestiert der Prager Schriftsteller und Kunstsammler Johann Ferdinand von Schönfeld den Wienern ein großes Interesse an Musik:

»Wien und Prag haben itzt so viele Schäzer, Freunde und Verehrer der Tonkunst - eben so große Meister und Dilettanten, daß es nun wirklich zum Bedürfniß geworden ist, von allen diesen ein instruktives Verzeichniß zu haben; - denn, wer sollte es wohl glauben, daß die Zahl derselben in Wien sich über 200 Menschen belaufe, die sich gleichsam vereinigt zu haben scheinen, die Ehre der Tonkunst bei uns auf den höchsten Grad zu bringen. ${ }^{6}$

Musikalische Veranstaltungen waren in Wien in privatem Rahmen weit verbreitet und Klavier- und Kammermusik hatten über die gesellschaftlichen Hierarchien hinweg einen wichtigen Stellenwert. Allerdings scheint sich diese Musikbegeisterung, anders als in Paris oder London, kaum in der Form von öffentlichen Konzertreihen manifestiert zu haben. Eine öffentliche Konzertkultur entwickelte sich nur sehr zaghaft, und noch Anfang des I9. Jahrhunderts lässt sich in Wien eine auffallende Zurückhaltung bei der Einrichtung von Konzertreihen beobachten. Interessant erscheint mithin auch, dass gerade die Sinfonien Haydns, Mozarts und Beethovens in einem Umfeld entstanden, das ein institutionalisiertes Konzertleben noch kaum kannte. Stefan Kunze spitzt dies in Bezug auf die drei Komponisten zu: »Keine der Sinfonien Haydns, Mozarts und Beethovens ist ja zu Lebzeiten der Komponisten in Wien im Rahmen etablierter Konzertreihen aufgeführt worden. « 7 Hier müsste allerdings differenziert werden, was eine >etablierte Konzertreihe ‘ tatsächlich bedeutet und was unter `Öffentlichkeit` verstanden wird.

Die Sinfonien Mozart schrieb, verglichen mit früheren Schaffensperioden, in seinen zehn Wiener Jahren ab I78 a auffallend viel Klaviermusik. Auch Beethovens CEuvre kann zu Beginn seiner Wiener Zeit als klavierbetont bezeichnet werden. Eduard Hanslick beschreibt im Rückblick die karge sinfonische Konzertlandschaft Wiens in der Zeit

5 Muzio Clementi: Mozart's celebrated Spmphony, THe Jupiter, newly adapted for the Piano-Forte, with Accompaniment for a Flute, Violin, and Violoncello, ad libitum, London I823.

6 Johann Ferdinand von Schönfeld ?]: Vorwort, in: Jahrbuch der Tonkunst von Wien und Prag, Wien I796, o. S.

7 Stefan Kunze: Die Sinfonie im I8. Jahrhundert. Von der Opernsinfonie zur Konzertsinfonie, Laaber I993 (Handbuch der musikalischen Gattungen, Bd.r), S. 289. 
Beethovens: »Was große sinfonische und chorische Wirkungen seien, hatte man in Wien in lauter kleiner Musik beinahe vergessen. ${ }^{8}$ Reine Instrumentalmusik erklang primär privat - oder sie war gekoppelt an das Burgtheater und das Theater am Kärntnertor. Während der Fastenzeit und im Advent durften dort keine Bühnendramen gespielt werden, sodass ab 1745 für diese Karenzzeiten einige Konzerte als Ersatzunterhaltung angeboten wurden. ${ }^{9}$ Nach und nach gelangten auch Sinfonien in die Programme, die sich sonst vor allem durch ein buntes musikalisches Gemisch auszeichneten, welches Vokales aus Opern und Oratorien nebst solistischen Darbietungen enthielt.

Mozarts erste öffentliche Beteiligung an einem privat organisierten Konzert im Theater am Kärntnertor datiert auf den 3. April I78ı. Mozart schreibt am Tag darauf seinem Vater: »ich spielte in der accademie der Witwen im kärtner thor Theater. - ich musste wieder Neuerdings anfangen, weil des applaudiren, kein Ende war. ${ }^{\mathrm{IO}}$ Kurze Zeit später kündigt ein Anschlagzettel an: »eine Sinphonie von der Komposition des Herrn Ritters Wolfgang Amadi Mozart «. ${ }^{\text {II }}$ Und dieser rapportiert seinem Vater nach der ersten Probe, »daß die Sinfonie Magnifique gegangen ist, und allen Succés gehabt hat «. ${ }^{\mathrm{I2}}$ Es handelte sich bei dieser Sinfonie wahrscheinlich um die Sinfonie Nr.3I (KV 297), die sogenannte Pariser Sinfonie.

Die Rahmenbedingungen für die Gattung der Sinfonie waren allerdings generell nicht ideal. Betrachtet man den Zeitraum zwischen I790 und ı80o, so beläuft sich die Zahl der aufgeführten Mozart-Sinfonien laut Wyn Jones auf gerade einmal zwölf, das heißt auf ungefähr eine jährlich oder rund ıo \% aller öffentlich in Wien gespielten Sinfonien. Die Jupiter sucht man vergebens darunter. Haydn kommt auf $50 \%{ }^{\text {I3 }}$

$\begin{array}{lcl}\begin{array}{l}\text { Sinfonieaufführungen } \\ \text { total }\end{array} & \begin{array}{l}\text { dav } \\ \text { von }\end{array} \\ \text { I790 } & \text { I4 } & \text { O } \\ \text { I79I } & \text { I5 } & \text { O } \\ \text { I792 } & 3 & 2 \\ \end{array}$

8 Eduard Hanslick: Geschichte des Concertwesens in Wien, Wien I869, S. I43.

9 Vgl. Martin Eybl/Elisabeth Fritz-Hilscher: Vom Barock zur Wiener Klassik (circa I740-I790/I80o), in: Wien Musikgeschichte. Von der Prähistorie bis zur Gegenwart, hg. von Elisabeth Fritz-Hilscher und Helmut Kretschmer, Wien 20II, S. 213-270, hier S. 230.

10 Brief W. A. Mozarts an seinen Vater vom 4. April I78I, zit. nach: Mozart. Briefe und Aufzeichnungen. Gesamtausgabe, Kassel 2005, Bd.3, S. IоI.

11 Wolfgang Amadeus Mozart. Neue Ausgabe sämtlicher Werke, Serie x: Supplement: Die Dokumente seines Lebens, hg. von Otto Erich Deutsch, Kassel I96ı, S. I73.

12 Brief W. A. Mozarts an seinen Vater vom II. April I78I, zit. nach: Mozart. Briefe und Aufzeichnungen. Gesamtausgabe, Bd.3, S. Io6.

13 Alle Zahlen nach David Wyn Jones: The Symphony in Beethoven's Vienna, Cambridge 2006, S. 52.

14 Nach dem Tod Kaiser Franz Josephs II. setzte eine wirtschaftliche und künstlerische Stagnation ein. 


$\begin{array}{lll}\text { I793 } & \text { IO } & \text { O } \\ \text { I794 } & 6 & \text { O } \\ \text { I795 } & \text { I2 } & \text { O } \\ \text { I796 } & \text { I5 } & 3 \\ \text { I797 } & 6 & 3 \\ \text { I798 } & \text { I5 } & \text { O } \\ \text { I799 } & 6 & 0 \\ \text { I800 } & \text { I4 } & 3\end{array}$

Während Wyns Angaben keine Quelle nennen, werden in der Allgemeinen musikalischen Zeitung von I80o die administrativen Hindernisse und die hohen Kosten für größere instrumentale Orchesteraufführungen als Hürden thematisiert:

»Im allgemeinen liebt man die Akademien hier nicht sehr, weil man schon zu oft getäuscht wurde, sowohl von fremden als einheimischen Spekulanten, worunter mancher brave Künstler leiden muß, wenn er nicht viel Protektion hat. Man erschwert ihm schon die Erlaubniß genug und dann sind noch die Kosten sehr bedeutend.«I5

In den Vaterländischen Blättern von I808 liest man:

»So viel jedoch für diese sogenannte Kammermusik gethan wird, so wenig Gelegenheit biethet sich für das volle Orchester, für Simfonien, Conzerte, Oratorien ec. dar. [Es] ist in diesem Fache nichts Solides, wenigstens nichts Dauerhaftes stabilirt worden. $\varkappa^{\mathrm{I}}$

Der Grund für diesen Umstand wird gleich anschließend angefügt:

»Wenn man erwägt, daß mit allem Uebrigen, auch die Preise zu großen Conzerten nöthigen BlaseInstrumentisten [...] beträchtlich gestiegen sind; daß die Bezahlung der Kopiaturen sich um das Doppelte, und jene der Wachskerzen sich um das Dreyfache erhöht hat; wenn man endlich weiß, daß die Einwohner von Wien, indessen sie Geist und Geschmack kultiviren, den Gaumen nicht gerne ganz vergessen, und es vorhin gewöhnlich war, bey großen Conzerten, wenn sie Abends gegeben wurden, das Orchester und die Zuhörer mit Erfrischungen zu neuem Eifer und neuer Aufmerksamkeit zu stärken; so wird man in der gegenwärtigen Theurung keinen unwahrscheinlichen Grund finden, warum die Gelegenheiten, die Musik im Großen auszuüben, und zu genießen, nicht so häufig sind, als man es, nach dem hier so allgemein herrschenden Geschmacke dafür, vermuthen sollte. «7

Um schon nur den Aufwand für die Beleuchtung zu illustrieren, sei Hanslick erwähnt, der ein - allerdings außergewöhnliches - Konzert von I8I2 in der eigens hergerichteten »k. k. Winterreitschule« erwähnt, die »von 5000 Wachskerzen erleuchtet« war. ${ }^{18}$

[o. A.]: Kurze Uebersicht des Bedeutendsten aus dem gesamten jetzigen Musikleben in Wien, in: Allgemeine musikalische Zeitung (AmZ) 3 (I800/or), Sp. 4I-5I sowie 65-69, hier Sp.50.

16 [o. A.]: Uebersicht des gegenwärtigen Zustandes der Tonkunst in Wien, in: Vaterländische Blätter I (ז808), S.39-44, hier S. 40.

17 Ebd., S.4I.

18 Hanslick: Geschichte des Concertwesens in Wien, Wien I869, S. I45. 
Die Jupiter - Abschriften, Orchesterstimmen, Drucke Die Wiener Rezeption der Jupiter fällt also in eine Zeit, in der die Stadt nur ein sehr eingeschränktes öffentliches Konzertleben hatte. Frühe Abschriften der Orchester-Stimmen deuten aber darauf hin, dass die Sinfonie schon zu Mozarts Lebzeiten gut bekannt war. Die breite Streuung der Manuskripte stellt Kunzes Folgerung, dass die Kenntnis des Werks sich wohl vorerst auf einen engen personellen und geografischen Kreis um Mozart beschränkte, ${ }^{\text {I9 }}$ zumindest in Frage.

In der ehemaligen Bibliothek des Fürsten von Oettingen-Wallerstein in Bayern findet sich eine Abschrift mit dem Titelvermerk »Di Wolfg: Amad: Mozart, Maestro di Capella in attuale Servicio di sua Maesta I'Imperatore ${ }^{20}{ }^{20}$ Dieser Titel (mit dem »attuale «) deutet darauf hin, dass Mozart bei der Kopierarbeit noch gelebt hat.

Ein weiteres Indiz für die Bekanntheit und gleichzeitig auch Beliebtheit der Sinfonie zeigt sich im Umstand, dass der erste Stimmensatz beim Verleger Johann André in Offenbach im Jahr I793, also bereits fünf Jahre nach der Entstehung, gedruckt wurde. Drucke waren an kommerzielle Interessen gebunden, und so ist anzunehmen, dass die Sinfonie bereits vor dem Druck in Form von Handschriften in Umlauf war und erst eine gewisse Bekanntheit zur Veröffentlichung Anlass gab. Diese Tatsache scheint umso bemerkenswerter, als dass keine institutionellen Konzertreihen in Wien existierten, welche einer Verbreitung förderlich gewesen wären. Beethoven hätte also ohne weiteres Zugang zum Stimmenmaterial oder einer handschriftlichen Partitur haben können. Der MozartExperte H. C. Robbins Landon geht davon aus, dass gedruckte Klavier-Arrangements noch vor den Orchesterstimmen in Umlauf gesetzt wurden. ${ }^{2 \mathrm{I}}$ Das würde bedeuten, dass die Jupiter nicht nur in Form von Orchesterstimmen oder Partituren verfügbar war, sondern auch als Klavierbearbeitung den Weg zu einem potentiellen Publikum, und somit auch zu Beethoven, hätte finden können.

Klavieradaptionen Mozart schreibt bei seiner Ankunft in Wien I78I an seinen Vater:

"Die Wiener sind wohl Leute die gerne abschiessen - aber nur am Theater. - und mein Fach ist zu beliebt hier, als daß ich mich nicht Souteniren [verteidigen] sollte. Hier ist doch gewis das Clavierland! $\ll^{22}$

19 Vgl. Stefan Kunze: Mozart. Jupiter-Sinfonie, München ı988, S. г3.

20 Zit. nach Robbins Landon: Zum vorliegenden Band, S. vir.

21 Vgl. ebd., S. viı.

22 Brief W. A. Mozarts an seinen Vater vom 2. Juni I78I, zit. nach Mozart. Briefe und Dokumente - OnlineEdition, hg. von der Internationalen Stiftung Mozarteum, Salzburg, http://dme.mozarteum.at/briefe (2I. Januar 20I9). 
Die Verbreitung der Jupiter als Klavieradaption scheint plausibel. In Wien gab es »gegen Ende des 18. Jahrhunderts schätzungsweise sechstausend Amateurpianisten und etwa dreihundert professionelle Klavierlehrer [...] - mehr als Ärzte.«23 I802 bewarb der Verlag Johann Traeg eine Bearbeitung der Jupiter-Sinfonie für 2 Klaviere durch Emanuel Aloys Förster. ${ }^{24}$ In den Vaterländischen Blättern von I8o8 wird berichtet: »In dieser großen Residenz [Wien] wird man wenige Häuser finden, in denen nicht an jedem Abende diese oder jene Familie sich mit einem Violin-Quartet, oder einer Klaviersonate unterhielte «. 5 Die große Nachfrage nach Klavier- und Kammermusikliteratur spiegeln auch die lokalen Verlagskataloge und -anzeigen wider. In der Allgemeinen musikalischen Zeitung wird I82I ein Arrangement von Breitkopf\& Härtel zu vier Händen ausführlich rezensiert und mit einer älteren Ausgabe verglichen. ${ }^{26}$ Auch wenn sowohl der Verlag wie auch die Zeitung in Leipzig ansässig waren, spiegelt die Besprechung ein allgemeines öffentliches Interesse an der Jupiter, welches auch für Wien angenommen werden dürfte.

Orchestrale Aufführungen Mögliche Aufführungen für die Jupiter in Wien sind bis I8oo nicht belegbar. Im April gleichen Jahres organisiert Beethoven seine erste Akademie und programmiert: »Eine grosse [sic] Symphonie von weiland Herrn Kapellmeister Mozart. « ${ }^{27} \mathrm{Ob}$ es sich tatsächlich um die Jupiter handelt, muss offenbleiben. Der Wiener Korrespondent der Leipziger Allgemeinen Zeitung berichtet: »Endlich bekam doch auch Herr Beethoven das Theater einmal, und dies war wahrlich die interessanteste Akademie seit langer Zeit. ${ }^{28}$ Bei dieser Gelegenheit dirigierte Beethoven seine I. Sinfonie, die auf dem Anschlagzettel wie folgt angekündigt wurde: »Eine neue grosse Symphonie mit vollständigen [sic] Orchester, komponirt von Herrn Ludwig van Beethoven. « ${ }^{29}$ Es fehlen also genau für die Zeit vor ı8oo, die Entstehungszeit der I. Sinfonie, Nachweise, welche dokumentieren könnten, dass Beethoven die Jupiter mit Orchester gehört hat. Das Fehlen von Berichten über öffentliche Konzerte in Wien mag darin begründet sein, dass die Presse nicht sonderlich an Privatveranstaltungen interessiert war. Die Wiener Zeitung war

Jan Caeyers: Beethoven. Der einsame Revolutionär. Eine Biographie, München 2012, S. I29.

Alexander Weinmann: Verlagsverzeichnis Johann Traeg (und Sohn), Wien I973 (Beiträge zur Geschichte des Alt-Wiener Musikverlages, Reihe 2, Bd.r6), S.32.

[o. A.]: Uebersicht des gegenwärtigen Zustandes der Tonkunst in Wien, S.39; später auch zit. bei Hanslick: Geschichte des Concertwesens in Wien, S. I44.

AmZ 23 (I82I), Sp. 847 f.

Caeyers: Beethoven, S. 227.

[o. A.]: Kurze Uebersicht des Bedeutendsten aus dem gesamten jetzigen Musikleben in Wien, Sp.49, später auch zit. bei Hanslick: Geschichte des Konzertwesens in Wien, S. I27.

29 Caeyers: Beethoven, S. 228. 
eine Hofzeitung und entsprechend ausgerichtet. Erst ab den I79oer-Jahren »bricht die Wiener Zeitung etwas häufiger ihr hartnäckiges Schweigen über Musik.«30 Konzertberichte finden sich trotzdem nur wenige. Den Beruf des Musikkritikers im heutigen Sinne gab es noch nicht. Der Philologe, Archäologe und Mozart-Biograf Otto Jahn erwähnt bereits I856 die Schwierigkeit, Quellen zum Musikleben in Mozarts Wien zu finden ${ }^{3 \mathrm{I}}$ und Hanslick doppelt 1869 nach:

»Otto Jahn hat guten Grund, in der Vorrede zu seiner Mozartbiographie über die spärlichen Quellen des älteren Musiklebens in Wien zu klagen. Namentlich über die Anfänge des Concertwesens und dessen Details nicht nur im vorigen, sondern noch in den ersten fünfzehn Jahren dieses Jahrhunderts sind wir sehr lückenhaft unterrichtet. «32

Private Aufführungen Für die Fastenzeit (April) I804 ist in Wien eine Aufführung der Jupiter in privatem Rahmen beim Bankier Joseph Baron von Würth dokumentiert. Würth, ein Musikliebhaber und Mäzen, konnte es sich leisten, an Sonntagvormittagen Konzerte zu organisieren. Gerne ließen sich die berühmtesten Virtuosen in seinem gastfreundlichen Haus hören. Dabei musizierte ein Orchester, das mehrheitlich mit Dilettanten besetzt war. Nur geladene und handverlesene Gäste wurden zu den hochwertigen Konzerten eingeladen. Die Berichterstattung in der Allgemeinen musikalischen Zeitung lautet: »Von den Sinfonieen erwähne ich nur die unübertrefflichen Mozartischen aus C dur und G moll; selbst das schwere und fugirte Finale der ersten wurde mit Feuer und Präzision ausgeführt.«33 Da die anderen C-Dur-Sinfonien Mozarts (KV 73, 96, I62, 200,338 und 425/Linzer) keinen fugierten Satz aufweisen, muss es sich dabei um die Jupiter, respektive damals eben noch die $>$ Sinfonie mit der Schlussfuge $<$, handeln.

Nur Tage vorher, im Februar I804, hatte Beethoven seine Eroica (Sinfonie Nr. 3 Es-Dur op. 55) in einer solchen Privatveranstaltung bei Würth dirigiert. ${ }^{34}$ Die Wahrscheinlichkeit ist also groß, dass die enge Beziehung zum Hause Würth dazu führte, dass Beethoven die Jupiter beim oben erwähnten Konzert gehört haben konnte. Zu diesem Zeitpunkt war auch sein Gehör noch dazu imstande. Würth programmierte die Jupiter erneut im Advent I804 $4^{35}$ und damit prominent zum Saisonauftakt. Erneut berichtet die Allgemeine musikalische Zeitung: Otto Jahn: W. A. Mozart, Leipzig I856, S. XxII. Hanslick: Geschichte des Concertwesens in Wien, S. XIII.

33 [o. A.]: Sonntagskonzert des Herrn von Würth, in: AmZ 6 (I804), Sp. 467 f., hier Sp. 468.

34 Hanslick: Geschichte des Concertwesens in Wien, S. 76.

35 Vgl. Mary Sue Morrow: Concert Life in Hapdn's Vienna. Aspects of a Developing Musical and Social Institution, Stuyvesant, NY I989, S. 402. 
»Für dieses Jahr geschah die Eröffnung mit der herrlichen Mozartschen Sinfonie aus C dur, welche mich bey jeder Aufführung von neuem begeistert. Das letzte fugierte Stück ward [...] mit Feuer und Präcision vorgetragen. $\aleph^{36}$

Es liegt nicht fern anzunehmen, dass Beethoven auch bei dieser Veranstaltung zugegen war.

Die nächsten öffentlichen Aufführungen der Jupiter in Wien finden sich in den Programmen der Liebhaber-Konzerte. Im November I807 berichtet der Wiener Korrespondent der Allgemeinen musikalischen Zeitung: »Man gab [...] Mozarts Riesensinfonie aus C mit dem fugierten Finale - diesen Triumph der Instrumentalmusik, und bisher noch unübertroffen! «37 Zwei mögliche, wenn auch nicht zweifelsfrei belegbare Aufführungen bei den Liebhaber-Konzerten datiert David Wyn Jones auf den 3. Januar und 6. März I808..$^{8}$

Zu dieser Zeit scheint es in anderen Städten wie Leipzig bereits eine Aufführungstradition der Jupiter gegeben zu haben. Dies zeigt ein Konzertbericht in der Allgemeinen musikalischen Zeitung aus dem Jahr ı808. Der unbekannte Verfasser unterstreicht die Beliebtheit der Sinfonie und erwähnt, dass sie mindestens jedes Jahr zur Aufführung komme:

«Noch besser, und wirklich meisterlich gelang die klassische Mozartsche [Sinfonie] aus $\mathrm{C}$ dur, mit der Schlussfuge - ein so erklärtes Lieblingsstück der hiesigen Kunstfreunde, dass wir sie ihnen kein Jahr vorenthalten. 39

Öffentliche Aufführungen Im Mai I8II taucht eine Mozart'sche C-Dur-Sinfonie in einem Programm der Tonkünstler-Societät in Wien auf. Ein Bericht der Allgemeinen musikalischen Zeitung erwähnt für den 8. Mai eine »declamatorische und musikalische Abend-Unterhaltung. Die vorkommenden Stücke waren: Erste Abteilung I) Symphonie (C dur) von Mozart, aber nur das erste Stück. «40 Ob es sich um die Jupiter handelte, kann nur vermutet werden.

Hanslick wird später auf den Umstand hinweisen, dass die Tonkünstler-Societät zu dieser Zeit offensichtlich wiederholt dasselbe Repertoire (Haydns »Cantaten«, gemeint sind seine Oratorien) spielte und die Sinfonien vernachlässigt wurden.

"Indem diesen vielbeschäftigten Fachmusikern das Interesse ihrer Pensionscasse doch noch wichtiger war als das künstlerische, kam in ihre Productionen nur zu bald ein handwerksmäßiger Schlendrian. Der Erfolg der beiden Haydn'schen Cantaten, mit denen durch Jahrzehnte auszulangen war, ließ

36 [o. A.]: Nachrichten [Konzert bei Würth in Wien], in: AmZ 7 (I805), Sp. 242 f., hier Sp. 242.

37 [о. A.]: Nachrichten [Liebhaberkonzerte in Wien], in: AmZ ıо (ı8०8), Sp. I84f.

38 Vgl. Jones: The Symphony in Beethoven's Vienna, S. I27f.

39 [o. A.]: Konzert in Leipzig. Neujahr bis Ostern, in: AmZ Io (I808), Sp. 48I-496, hier Sp. 495.

40 [o. A.]: [Konzert im Kärntnertor-Theater], in: AmZ I3 (I8II), Sp.360 f., hier Sp.360. 
die ,Societät‘ an eine Erneuerung ihres Repertoirs gar nicht denken; sie vernachlässigte insbesondere die reine Instrumentalmusik gänzlich. Man liest in den ersten I5 Jahren dieses Jahrhunderts häufig die Klage, daß in Wien die Aufführung einer Sinfonie eine Seltenheit geworden und die Instrumentalcompositionen Haydn's und Mozart's in Vergessenheit gerathen.« ${ }^{4 \mathrm{~T}}$

Diese Lücke wurde mit neu organisierten Veranstaltungen zu schließen versucht. I8ı2 wurden die »Gesellschafts-Concerte der österreichischen Musikfreunde«gegründet und I8I9 die »Concerts Spirituels« ins Leben gerufen. Ihre sogenannten »Übungskonzerte« sollten »vorzüglich[e] Symphonien« und »geistlich[e] Tonstücke berühmter Meister alter und neuer Zeit « auf hohem Niveau zur Aufführung bringen. ${ }^{42}$

Die statutarischen Absichten manifestierten sich auch in den Aufführungen von Mozarts Jupiter. Für die zehn Jahre von I8I7 bis zu Beethovens Tod I827 lassen sich sieben öffentliche Aufführungen der Jupiter in Wien belegen:

I8I7 Gesellschaft der Musikfreunde: »C dur-Symphonie«

I8I9 Concerts Spirituels: »C-Sinfonie«

ı820 Gesellschaft der Musikfreunde: »C-Sinfonie«

I822 Gesellschaft der Musikfreunde: »Sinfonie (mit der Fuge)《

I824 Gesellschaft der Musikfreunde: »C-Sinfonie «3

I825 (April) Gesellschaft der Musikfreunde: »die große in C dur mit der Fuge ${ }^{44}$

I827 Concerts Spirituels: »herrlich[e] Symphonie in C« ${ }^{45}$

Es gab also mindestens sieben weitere Gelegenheiten, bei welchen Beethoven die Jupiter im Konzert hätte erleben können, obschon seine fortschreitende Taubheit es ihm ab ı8ı2 wohl nahezu verunmöglichte, dass er sie hätte hören können. Allerdings ist Beethovens Verehrung für Mozart bekannt. Willi Reich und Paul Nettl zitieren ohne Quellenangabe den Zeitgenossen Anton Schindler, der erwähnt haben soll, dass Beethoven schon bei seinem ersten Besuch in Wien einzig vom Kaiser und von Mozart beeindruckt gewesen sei. ${ }^{46}$ Neben seinen eigenen Werken duldete Beethoven in seinen Programmen vor allem Haydn und Mozart, was auf die sehr gute Kenntnis und die Wertschätzung von Mozarts Euvre hindeutet. Ob für einen normalen Wiener Musikfreund die sieben öffentlichen

41 Hanslick: Geschichte des Concertwesens in Wien, S. I44.

42 Andrea Harrandt: Concerts spirituels, in: Oesterreichisches Musiklexikon, hg. von Rudolf Flozinger, Bd. I, Wien 2002, S. 283.

43 Hanslick: Geschichte des Concertwesens in Wien, I8I7: S. I56, I819: S. I89, I820 und I822: S. I58, I824: S. I59.

44 [o. A.]: Notizen, in: Der Sammler. Ein Unterhaltungsblatt I7 (I825), S. 235.

45 [o. A.]: Wien. Musikalisches Tagebuch vom Monat März, in: AmZ 29 (I827), Sp. 284.

46 Vgl. Paul Nettl: Beethoven und seine Zeit, Frankfurt a. M. I958, S. I9. Diese Aussage findet sich erst in der erweiterten dritten Auflage von Anton Schindler: Biographie von Ludwig van Beethoven, 3., neu bearb. und verm. Aufl., Münster i86o, Bd. I, S. I5. 
Aufführungen der Jupiter - verteilt auf acht Jahre - ausgereicht haben, um die Sinfonie als Orchesterwerk kennen und schätzen zu lernen, bleibt eine offene Frage.

Fazit Es scheint sehr wahrscheinlich, dass Beethoven die Jupiter als Orchesteraufführung in Wien zu Gehör bekam. Die Privataufführung bei Baron Würth, zu dem Beethoven einen engen Kontakt pflegte und bei dem er seine eigenen Werke zur (Ur-) Aufführung brachte, bot jedenfalls die Gelegenheit hierzu. Der Zeitpunkt dieser JupiterAufführung lag günstig. Beethovens Dirigat bei Würth stand unmittelbar bevor und sein Gehör war noch recht gesund. Allerdings war da Beethovens I. Sinfonie bereits geschrieben und aufgeführt worden. Der Kontakt mit der Jupiter bei Würth konnte also keinen Einfluss mehr auf Beethovens Kompositionsprozess seiner C-Dur-Sinfonie gehabt haben.

Den meisten musikinteressierten Wienern dürfte die Jupiter im Konzertsaal entgangen oder aufgrund der seltenen Wiederholungen als Orchesterstück nicht in Erinnerung geblieben sein. Das Wien Beethovens mit seinem beschränkten öffentlichen Konzertwesen war nicht der ideale Nährboden, auf welchem ein sinfonisches Repertoire ohne weiteres gedeihen konnte. Die Frage drängte sich auf, warum gerade diese Epoche als Geburtszeit und die Stadt Wien als Wiege der instrumentalen >Klassik^gelten. Eine Frage, die zu beantworten an dieser Stelle nicht nötig ist. Die Darstellung der Gegebenheiten rund um die Jupiter sollten lediglich den oft verklärten Blick auf die Aufführungssituation der Zeit illustrieren.

Zwischen der Rezeptionsgeschichte der Jupiter-Sinfonie in Beethovens Wien und ihrer Gegenwärtigkeit im heutigen Konzertbetrieb besteht eine große Diskrepanz. Sie zeigt, dass sich die Bekanntheit und vielleicht auch die Beliebtheit einzelner Kompositionen im Wandel der Zeit durchaus verändern können. Gründe mögen in der sich wandelnden Ästhetik, aufflackernden Moden oder Instrumentalisierungen durch Politik oder Marketing zu finden sein. Ein Blick in die heutigen Konzertprogramme zeigt jedenfalls, dass die Jupiter heute wohl mehr denn je als eines der »erklärten Lieblingsstücke« der Kunstfreunde rund um den Globus gelten kann. 


\section{Inhalt}

Vorwort 8

INTERPRETATION - BEGRIFF, METHODE, PRAXIS

Laure Spaltenstein Interpretation als treue Übersetzung. Zur Frühgeschichte eines vieldeutigen Begriffs I5

Kai Köpp Von der Quelle zur Methode. Zum Entwurf einer historischen Interpretationsforschung $\quad 28$

Manuel Bärtsch >Interpretation‘. Beethovens Sonate A-Dur op. IOI in der Sicht von Eugen d'Albert und Frederic Lamond

Sebastian Bausch Klavierrollen als Interpretationsdokumente. Ein Erfahrungsbericht als Leitfaden für Einsteiger $\quad 7 \mathrm{I}$

Camilla Köhnken Beethoven-Auslegung zwischen Liszts »Deklamationsstil« und Bülows »Vivisektionsversuchen«. Auf den Spuren Liszt'scher Interpretationsideale in Hans von Bülows instruktiver Edition der Klaviersonaten Beethovens $\quad 92$

Neal Peres Da Costa Carl Reinecke's Performance of his Arrangement of the Second Movement from Mozart's Piano Concerto K. 488. Some Thoughts on Style and the Hidden Messages in Musical Notation

II4

Carolina Estrada Bascuñana Enrique Granados's Performance Style.

Visualising the Audible Evidence I5O

Lukas Näf Tempogestaltung in Weberns Sinfonie op. 2I I80

INTERPRETATION - AUFFÜHRUNGSGESCHICHTE

Christoph Moor "Ein so erklärtes Lieblingsstück der hiesigen Kunstfreunde«. Die Rezeptionsgeschichte der Jupiter-Sinfonie in Beethovens Wien

Luisa Klaus Objektive Bruckner-Interpretation? Zur Aufführung der Trio-Entwürfe für die Neunte Sinfonie I940 205

Chris Walton Von innen und von außen. Beethovens Neunte Sinfonie und die $>$ Wagner'sche< Dirigiertradition $\quad 2 \mathrm{I} 8$

Lena-Lisa Wüstendörfer Streit um Fidelio. Gustav Mahler und Felix Weingartner im Disput um Werktreue $\quad 238$

INTERMEZZO

Robert Levin Turning Point to Musical Modernity. Beethoven as Executor of the Legacy of C. P. E. Bach. Concert Lecture 249

INTERPRETATION - INSTRUMENTE, ANALYSE, EDITION

Martin Skamletz «Man hat diese Erweiterung des Tonumfanges seit ein paar Jahren an den Tasteninstrumenten sehr weit getrieben.« Der Umgang mit Grenzen beim späten Mozart und beim frühen Beethoven $\quad 263$ 
Stephan Zirwes Analyse und Interpretation. Adolph Bernhard Marx' Beethoven-Analysen 29I

Michael Ladenburger Was können wir aus Originalhandschriften von Beethoven für eine angemessene Interpretation lernen? 30I

Federica Rovelli Die Skizzenbuch-Ausgaben und ihre mögliche digitale Zukunft 3 I7

Johannes Gebauer Interpretationspraktische Stemmatik. Philologische Methoden in der Interpretationsforschung am Beispiel annotierter Notenausgaben von Rodes 24 Capricen und Beethovens Violinkonzert

John Rink Chopin Copying Chopin 349

Tomasz Herbut Alexander Goldenweiser und Beethovens Sonate op. IIo- eine Spurensuche 366

INTERPRETATION - KREATIVE ANEIGNUNG

Thomas Gartmann Beethoven als sein eigener Interpret. Gedanken zur Bearbeitung der Klaviersonate op. I4/ז für Streichquartett

Ivo Haag Die Sinfonien von Johannes Brahms - (auch) Klaviermusik?

Michael Lehner Das Orchester auf dem Klavier. Welte-Klavierrollen von Gustav Mahler und Richard Strauss als interpretationsanalytische Quellen

Roger Allen “That Is What Music Really Is". Richard Wagner's Reception of Beethoven's Piano Sonata in A Major Op. IOI $43 \mathrm{I}$

Daniel Allenbach Eine heroische< Neunte? Dmitri Schostakowitschs Neunte Sinfonie im Vergleich mit Ludwig van Beethoenvs Sinfonien Nr.3 und 9 44I

Simeon Thompson Beethoven und der Zweite Weltkrieg in der künstlerischen Reflexion der Nachkriegszeit. Stanley Kubricks A Clockwork Orange und Rolf Liebermanns Leonore 40/45 456

Michelle Ziegler Rettungsversuch im Jubiläumsjahr. Mauricio Kagels Aufarbeitung der Beethoven-Rezeption in der Ludwig van-Werkgruppe (I970) 465

Leo Dick Über den späten Beethoven zur >Postidentität،. Die Suche nach liminalen Räumen im gegenwärtigen Musiktheater am Beispiel von Matthias Rebstocks Berliner Produktion Büro für postidentisches Leben $\quad 476$

Elizabeth Waterhouse Choreographic Re-mix. William Forsythe's Trio (I996) and Beethoven's String Quartet No. I5 in a Minor Op. I32 487

László Stachó "Gradus ad Parnassum".

The Purgatory of Instrumental Technique

Namen-, Werk- und Ortsregister 522

Die Autorinnen und Autoren der Beiträge 


\section{Rund um BeEthoven \\ Interpretationsforschung heute • \\ Herausgegeben von Thomas}

Gartmann und Daniel Allenbach 


\section{MUSIKFORSCHUNG DER Hochschule der KÜnste Bern Herausgegeben von Martin Skamletz und Thomas Gartmann Band 14}


0 Dieses Buch ist in gedruckter Form im Dezember 2019 in erster Auflage in der Edition Argus in Schliengen/Markgräflerland erschienen. Gestaltet und gesetzt wurde es im Verlag aus der Seria und der SeriaSans, die von Martin Majoor im Jahre 2000 gezeichnet wurden. Gedruckt wurde es auf Eos, einem holzfreien, säurefreien, chlorfreien und alterungsbeständigen Werkdruckpapier der Papierfabrik Salzer im niederösterreichischen Sankt Pölten. Das Vorsatzpapier Caribic cherry wurde von Igepa in Hamburg geliefert. Rives Tradition, ein Recyclingpapier mit leichter Filznarbung, das für den Bezug des Umschlags verwendet wurde, stellt die Papierfabrik Arjo Wiggins in Issy-les-Moulineaux bei Paris her. Das Kapitalband mit rot-schwarzer Raupe lieferte die Firma Dr. Günther Kast aus Sonthofen im Oberallgäu, die auf technische Gewebe und Spezialfasererzeugnisse spezialisiert ist. Gedruckt und gebunden wurde das Buch von der Firma Bookstation im bayerischen Anzing. Im Internet finden Sie Informationen über das gesamte Verlagsprogramm unter www.editionargus.de, zum Institut Interpretation der Hochschule der Künste Bern unter www.hkb.bfh.ch/interpretation und www.hkb-interpretation.ch. Die Deutsche Nationalbibliothek verzeichnet diese Publikation in der Deutschen Nationalbibliografie; detaillierte bibliografische Daten sind im Internet über www.dnb.de abrufbar. (c) der zeitgleich erschienenen digitalen Version: die Autorinnen und Autoren, 20I9. Dieses Werk ist lizenziert unter einer Creative Commons Namensnennung-Nicht kommerziell 4.0 International Lizenz (CC BY-NC 4.o). DoI: https://doi.org/I0.26045/kp64-6I78 ISBN 978-3-93I264-94-9 